\title{
The Effect of An Educational Program on Nurses' Practices Regarding The Implementation of Patient Care and Safety Measures During Nasogastric Tube Feeding in The Critical Care Units in Syria
}

Yaser Adnan Abo Jeesh

College of Applied Medical Sciences-Alzaiem Alazhari University, Sudan Elham Fath-Elalium Mohammed Khalid

College of Medicine, Alzaiem Alazhari University, Sudan

Ishraqa Mohammed Ahmed Elbashier

College of Applied Medical Sciences-Alzaiem Alazhari University, Sudan

Doi:10.19044/esj.2021.v17n29p59

Submitted: 14 July 2021

Accepted: 09 August 2021

Published: 31 August 2021
Copyright 2021 Author(s)

Under Creative Commons BY-NC-ND 4.0 OPEN ACCESS

Cite As:

Jeesh Y.A.A., Khalid E.F-E.M. \& Elbashier I.M.A. (2021). The Effect of An Educational Program on Nurses' Practices Regarding The Implementation of Patient Care and Safety Measures During Nasogastric Tube Feeding in The Critical Care Units in Syria. European Scientific Journal, ESJ, 17(29), 59.

https://doi.org/10.19044/esj.2021.v17n29p59

\section{Abstract}

The intensive care unit (ICU) is considered a high- risk area in the hospital because of the severity of injuries and the high liability of occurrences associated with ICU. Critically ill patients are at high risk for actual or potential life-threating health problems. Moreover, critical care nurses must develop their knowledge, skills, and safe practice in acute areas where patients require more detailed observation or intervention to deliver safe and effective care. Objectives: This study aimed to assess the effect of an educational program on nurses' practices regarding the implementation of patient care and safety measures during enteral feeding. Design: The quasi-experimental design was used in this study. Methods: The study was conducted from June to September 2020. All registered nurses who were working in the intensive care units during the period of data collection comprised the sample. Based on the nurses' needs regarding the condition of their competencies during the initial assessment, we developed and designed the educational program. This program consists of two parts: theory and practical. The program contains 
workshop (8 hours) per day and educational pamphlet handout. The workshop consists of PowerPoint presentations, group discussions, and training scenarios. Results: This study was carried out with 48 nurses working in the previous intensive care units. The nurses' score were improved after the program regarding to nurses' age, qualification, and experience. The overall study found a statistically significant relationship of this procedure before and after the implementation of the educational program $(p<0.001)$. Conclusion: There has been remarkable and variation improvement of nurses performance after they received the education program. The results show that a statistically significant difference was found before and after implementing the program regarding the nurses' age, qualification, and experience. It is recommended to pay more attention to these nurses by helping them to improve their knowledge and practices in all nursing interventions. Furthermore, it is necessary to improve nurses' awareness regarding the quality of care and patient safety. It was also found that the poor knowledge and practice and its complications have a negative impact on the quality of care and patient safety.

Keywords: Intensive Care Unit, Patient, Care, Safety

\section{Introduction}

The intensive care unit (ICU) is a specially staffed and equipped, separate and self-contained, section of the hospital for the management of patients with life threatening or potentially life-threatening conditions (Australia \& Zealand, 2010; Rungta Govil et al., 2010).

Over the past decade, there has been increasing attention to issues surrounding patient safety and human error in health care and specifically in the critical care area. Adverse events have often been used as indicators of quality and safety in health service delivery. Patient safety requires continuous monitoring and search for new strategies (Alhawsawi, Wazzan et al., 2017; Alotaibi \& Federico, 2017; Organization, 2020).

Critical care nurses must develop their knowledge, skills, and safe practice in acute areas where patients require more detailed observation or intervention to deliver safe and effective care (Marshall Bosco et al., 2017).

The patients in intensive care unit vary from most other hospital patients because they have bounded ability to participate in medical care, and there is scarcity of physiologic reserve to bear additional injury. On average, 1.7 medical errors are experienced each day in the intensive care unit, and many patients endure a potentially life-threatening error during their stay (Camiré Moyen et al., 2009).

Critically ill patients are often unable to eat because of endotracheal intubation and the need for mechanical ventilation. In addition, early nutritional support improves patient outcomes and enhanced recovery from 
illness (Wiegand, 2013). Enteral nutrition should be prescribed whenever oral intake is inadequate for patients who have functional gastrointestinal tract. Although total parenteral nutrition can be life-saving, it can generate a wide array of complications in itself (Boullata Carrera et al., 2017; Anderson, 2019).

Nursing care is the key to positive outcomes in patient requiring enteral feeding. Understanding the decision-making process for the use of this therapy along with the steps of feeding initiation, complications, prevention, and feeding termination gives nursing personnel the tools they need to deliver nutrition in a safe and cost-effective manner (Thompson, 1997; Boullata Carrera et al., 2017; Anderson, 2019).

\section{Complications of Enteral Feeding}

The complications or side effects of enteral nutrition can be classified according to etiology: mechanical, gastrointestinal or functional, metabolic and infectious complications. Critical care nurses must be alert to all of these potential complications to administer enteral nutritional therapy effectively and safely (Bower, 1990; Btaiche Chan et al., 2010; Vaithiswaran Srinivasan et al., 2010).

Mechanical complications can be divided into two types of complications; the first type occurs during tube insertion and the second occurs during the feeding process (Ciocon Silverstone et al., 1988; Strollo McClave et al., 2017).

Aspiration is the most dangerous mechanical complication associated with enteral feeding (Scolapio, 2007; Blumenstein Shastri et al., 2014). Critical care nurses play a vital role in early detection and prevention of aspiration (Persenius Larsson et al., 2006; Serna \& McCarthy, 2006).

Tube obstruction is a mechanical complication associated with nasogastric tube feeding. Nursing interventions to prevent or decrease tube obstruction entail the following: obtaining liquid medications when possible, flushing feeding tube before and after medication administration, and diluting feeding with water if it is dense and straining if necessary (Metheny Meert et al., 2007; Aziz \& Ali, 2020).

Tube displacement is another mechanical complication of nasogastric tube feeding. Tube displacement may be caused by excessive coughing, vomiting, tracheal suctioning, airway intubation and this can be managed by checking tube placement before administering feeding (Metheny Meert et al., 2007; Marshall Cahill et al., 2012).

Gastrointestinal complications include: nausea and vomiting, diarrhea, constipation, and delayed gastric emptying. Nausea and vomiting, associated with enteral feeding, can be caused by the following: tube migration into the esophagus, decreased absorption that lead to increase in the gastric residual 
volume, and hyperosmolar formula and excessive infusion of air (Gavi Hensley et al., 2008; Mooi \& Ncama, 2019).

Delayed gastric emptying, Distention, are another gastrointestinal complications associated with tube feeding (Ang Lim et al., 2020).

Diarrhea is a common gastrointestinal problem in any tube fed patient. It may be caused by drug therapy, hypoalbuminemia or high osmolarity of the formula, rapid infusion, bolus feeding, and bacterial-contaminated feeding which is considered as a significant cause of diarrhea (Blumenstein Shastri et al., 2014; McLaren \& Arbuckle, 2020).

Nursing interventions to reduce these complications include checking residuals and holding feeding for one hour and rechecking if high gastric residual is found, changing the patient position to the right side, evaluating the drug regimen, changing to high fiber formula and assessing fluid balance, electrolyte levels, as well as checking formula temperature is a must, and maintaining the patients head of bed elevated at 30-60 minutes after feeding (Bourgault Ipe et al., 2007; Mooi \& Ncama, 2019; Ang Lim et al., 2020; Aziz \& Ali, 2020).

\section{Methods}

The quasi-experimental design was used in this study. This study was conducted to assess the effect of an educational program on nurses' practices regarding the implementation of patient care and safety measures during nasogastric feeding in the critical care units at Al-Assad University HospitalDamascus- Syrian Arab Republic. All registered nurses (48) with different age groups, different qualifications, and different experiences, who were working in the critical care units during the period of data collection, comprised the sample. All the official approvals were obtained from the relevant authorities.

\section{Tools}

The tool used in this study is divided into two parts: (1) demographic data of the nurses, age, qualification, and experience; (2) The observational checklist (developed by the researcher) to observe nurse's implementation of patient care and safety measures during nasogastric tube feeding procedure. A pilot study was conducted on 5 nurses not included in the study to test the clarity, applicability, and feasibility of the tool. Appropriate modifications were performed prior to data collection for the actual study.

\section{Data Collection}

Data collection was carried out from June 2020 to September 2020. The phases of data collection were: (1) All nurses were observed once initially, (2) the educational program was developed based on the nurses' needs, (3) implementing the program, and (4) reassessing the nurses. 


\section{Statistical Analysis}

The data were analyzed by using the Statistical Package for the Social Science (SPSS version 20.0). Significance of the obtained results was judged at the $5 \%$ level.

\section{Educational Program}

Educational program is part of the tools that were developed. Thus, the researcher designed and developed the educational program based on the nurses' needs regarding the condition of their competencies during the initial assessment.

The program consists of workshop (8 hours) per day (PowerPoint presentations and training sessions) and educational pamphlet handout.

\section{Results}

The total number of the studied nurses was 48 nurses. In relation to age, 17 nurses (more than 35\%) of the studied nurses were from 25-29 age group, 14 nurses (29.2\%) for age group from 20-24 and the same percentage for nurses with age group from 30-35, and the nurses who have more than 35 years were 3 nurses (6.3 \%). The mean of nurses' age was 28 years (Table 1).

In regard to their qualifications, the majority of the studied nurses, 38 nurses (about 80\%), have a diploma degree, 7 nurses (about 15\%) have a bachelor degree, and 3 nurses (about 6\%) have a master degree (Table 1).

In regard to their years of experience, 23 nurses (47.9\%) have less than 5 years of experience, while 17 nurses (35.4\%) have between five to ten years of experience, and 8 nurses (16.7 \%) have more than 10 years of experience. The mean of nurses’ experience was 6 years (Table 1).

Table 1. The characteristics of the studied nurses

\begin{tabular}{|c|c|c|}
\hline Demographic data & No. & $\mathbf{\%}$ \\
\hline Age of the nurse & & 29.2 \\
\hline $20-24$ & 14 & 35.4 \\
\hline $25-29$ & 17 & 29.2 \\
\hline $30-35$ & 14 & 6.3 \\
\hline Above 35 & 3 & $28.02 \pm 5.51$ \\
\hline Mean \pm SD. & \multicolumn{2}{|c|}{} \\
\hline Qualification of the nurse & 38 & 79.2 \\
\hline Diploma & 7 & 14.6 \\
\hline Bachelor & 3 & 6.3 \\
\hline Master & 23 & 37.9 \\
\hline Experience of the nurse in ICU & 17 & 16.7 \\
\hline Less than 5 years & 8 & \\
\hline 5- 10 years & \multicolumn{2}{|c|}{$6.27 \pm 4.53$} \\
\hline More than 10 years & \multicolumn{2}{|c|}{} \\
\hline Mean \pm SD. & & \\
\hline & & \\
\hline
\end{tabular}


Regarding the implementation of patient care and safety measures of the studied nurses, before the program, the results show that the nurses ignored and didn't implement most steps of this procedure in the correct way. However, the total steps of this procedure were twenty-three. The nurses didn't implement twelve steps correctly at all, while the percentage of the other eleven steps that was implemented correctly ranged from 2.1 to $41.7 \%$ (Table 2 ). The results show that there were variations of the nurses' improvement in all steps after the program, and the percentage of the nurses that implement the steps correctly ranged from $2.1-100 \%$. In addition, the nurses' scores were varied from 35-80 with mean of $50.69 \pm 10.35$ before the program to 54-89 with mean of $72.10 \pm 9.07$ after the program.

Table 2. The description and implementation of patient care and safety measures of the studied nurses according to nasogastric tube feeding items before and after the

educational program

\begin{tabular}{|c|c|c|c|c|c|c|c|c|c|c|c|c|c|c|c|c|}
\hline \multirow{4}{*}{ Feeding } & \multicolumn{16}{|c|}{ Frequency $(\mathrm{N}=48)$} \\
\hline & \multicolumn{8}{|c|}{ Before an educational program } & \multicolumn{8}{|c|}{ After an educational program } \\
\hline & \multicolumn{2}{|c|}{ Not done } & \multicolumn{2}{|c|}{$\begin{array}{c}\text { Done } \\
\text { incorrectly }\end{array}$} & \multicolumn{2}{|c|}{$\begin{array}{c}\text { Need more } \\
\text { practice }\end{array}$} & \multicolumn{2}{|c|}{$\begin{array}{c}\text { Done } \\
\text { correctly }\end{array}$} & \multicolumn{2}{|c|}{ Not done } & \multicolumn{2}{|c|}{$\begin{array}{c}\text { Done } \\
\text { incorrectly }\end{array}$} & \multicolumn{2}{|c|}{$\begin{array}{c}\text { Need more } \\
\text { practice }\end{array}$} & \multicolumn{2}{|c|}{$\begin{array}{c}\text { Done } \\
\text { correctly }\end{array}$} \\
\hline & No. & $\%$ & No. & $\%$ & No. & $\%$ & No. & $\%$ & No. & $\%$ & No. & $\%$ & No. & $\%$ & No. & $\%$ \\
\hline Assess the patient's gastric residual volume and content & 21 & 43.8 & 26 & 54.2 & 1 & 2.1 & 0 & 0.0 & 0 & 0.0 & 13 & 27.1 & 25 & 52.1 & 10 & 20.8 \\
\hline Listen to bowel sounds & 10 & 20.8 & 32 & 66.7 & 5 & 10.4 & 1 & 2.1 & 0 & 0.0 & 6 & 12.5 & 33 & 68.8 & 9 & 18.8 \\
\hline Confirm ETT/TT cuff inflation & 9 & 18.8 & 23 & 47.9 & 15 & 31.3 & 1 & 2.1 & 0 & 0.0 & 10 & 20.8 & 18 & 37.5 & 20 & 41.7 \\
\hline Assess the patient's need for suction & 11 & 22.9 & 25 & 52.1 & 12 & 25.0 & 0 & 0.0 & 1 & 2.1 & 9 & 18.8 & 23 & 47.9 & 15 & 31.3 \\
\hline Confirm NGT placement & 1 & 2.1 & 29 & 60.4 & 16 & 33.3 & 2 & 4.2 & 0 & 0.0 & 6 & 12.5 & 29 & 60.4 & 13 & 27.1 \\
\hline Prepare all necessary equipment & 0 & 0.0 & 24 & 50.0 & 21 & 43.8 & 3 & 6.3 & 0 & 0.0 & 4 & 8.3 & 21 & 43.8 & 23 & 47.9 \\
\hline $\begin{array}{l}\text { Assess feeding formula for color, odor, amount \& expiration } \\
\text { date }\end{array}$ & 8 & 16.7 & 35 & 72.9 & 5 & 10.4 & 0 & 0.0 & 1 & 2.1 & 6 & 12.5 & 21 & 43.8 & 20 & 41.7 \\
\hline Wash hands & 16 & 33.3 & 26 & 54.2 & 6 & 12.5 & 0 & 0.0 & 0 & 0.0 & 16 & 33.3 & 24 & 50.0 & 8 & 16.7 \\
\hline Wear gloves & 14 & 29.2 & 14 & 29.2 & 12 & 25.0 & 8 & 16.7 & 0 & 0.0 & 5 & 10.4 & 15 & 31.3 & 28 & 58.3 \\
\hline Put patient in the appropriate position & 0 & 0.0 & 24 & 50.0 & 17 & 35.4 & 7 & 14.6 & 0 & 0.0 & 1 & 2.1 & 29 & 60.4 & 18 & 37.5 \\
\hline Maintain privacy throughout the procedure & 21 & 43.8 & 25 & 52.1 & 2 & 4.2 & 0 & 0.0 & 4 & 8.3 & 19 & 39.6 & 25 & 52.1 & 0 & 0.0 \\
\hline Irrigate NGT with water & 10 & 20.8 & 33 & 68.8 & 5 & 10.4 & 0 & 0.0 & 0 & 0.0 & 10 & 20.8 & 34 & 70.8 & 4 & 8.3 \\
\hline Administer feeding by gravity. & 0 & 0.0 & 4 & 8.3 & 13 & 27.1 & 31 & 64.6 & 0 & 0.0 & 0 & 0.0 & 4 & 8.3 & 44 & 91.7 \\
\hline Avoid entering air throughout the procedure & 7 & 14.6 & 31 & 64.6 & 10 & 20.8 & 0 & 0.0 & 0 & 0.0 & 10 & 20.8 & 30 & 62.5 & 8 & 16.7 \\
\hline Re-irrigate NGT with water & 5 & 10.4 & 33 & 68.8 & 10 & 20.8 & 0 & 0.0 & 0 & 0.0 & 10 & 20.8 & 30 & 62.5 & 8 & 16.7 \\
\hline Wash tommy syringe if needed & 11 & 22.9 & 28 & 58.3 & 9 & 18.8 & 0 & 0.0 & 1 & 2.1 & 13 & 27.1 & 27 & 56.3 & 7 & 14.6 \\
\hline Reconnect tommy syringe to NGT & 0 & 0.0 & 2 & 4.2 & 26 & 54.2 & 20 & 41.7 & 0 & $\begin{array}{ll}2.1 \\
0.0\end{array}$ & 0 & 0.0 & 0 & 0.0 & 48 & 100 \\
\hline Discard disposable equipment & 2 & 4.2 & 35 & 72.9 & 11 & 22.9 & 0 & 0.0 & 0 & 0.0 & 2 & 4.2 & 30 & 62.5 & 16 & 33.3 \\
\hline Return feeding formula to the refrigerator & 0 & 0.0 & 28 & 58.3 & 16 & 33.3 & 4 & 8.3 & 0 & 0.0 & 3 & 6.3 & 21 & 43.8 & 24 & 50.0 \\
\hline Remove gloves & 14 & 29.2 & 14 & 29.2 & 12 & 25.0 & 8 & \begin{tabular}{|c|c|}
0.7 \\
16.7
\end{tabular} & 3 & \begin{tabular}{|l|l|}
6.3 \\
\end{tabular} & 9 & 18.8 & 13 & 27.1 & 23 & 47.9 \\
\hline Wash hands & 16 & 33.3 & 26 & 54.2 & 6 & 12.5 & 0 & 0.0 & 1 & $\begin{array}{ll}2.1 \\
\end{array}$ & 13 & 27.1 & 26 & 54.2 & 8 & 16.7 \\
\hline $\begin{array}{l}\text { Instruct the patient that the position should be maintained for } \\
\text { at least } 30-45 \mathrm{~m}\end{array}$ & 8 & 16.7 & 36 & 75.0 & 4 & 8.3 & 0 & 0.0 & 3 & 6.3 & 9 & 18.8 & 35 & 72.9 & 1 & 2.1 \\
\hline Record date, time. \& type \& amount of feeding formula. & 9 & 18.8 & 28 & 58.3 & 8 & 16.7 & 3 & 6.3 & 0 & 0.0 & 11 & 22.9 & 30 & 62.5 & 7 & 14.6 \\
\hline \multicolumn{17}{|l|}{ Total score of feeding (23-92) } \\
\hline Min.-Max. & \multicolumn{8}{|c|}{$35.0-80.0$} & \multicolumn{8}{|c|}{$54.0-89.0$} \\
\hline Mean \pm SD & \multicolumn{8}{|c|}{$50.69 \pm 10.35$} & \multicolumn{8}{|c|}{$72.10 \pm 9.07$} \\
\hline$t$ (p) & \multicolumn{16}{|c|}{$\left.<0.001^{*}\right)$} \\
\hline
\end{tabular}

\section{t: Paired t-test}

p: $\mathrm{p}$ value for comparing between Before and After *: Statistically significant at $\mathrm{p} \leq 0.05$

Based on the implementation of patient care and safety measures regarding the nurses' age groups, the total scores of this procedure is 92 . Regarding to nurses' age (20-24), the results show that the scores before the program varied from 49-59 with a mean and standard deviation of $53.71 \pm$ 2.78, while the nurses' score after the program varied from 74-79 with a mean and standard deviation of $77.57 \pm 1.60$. Regarding to nurses with 25-29 age group, the scores before the program ranged from $40-80$ with a mean and standard deviation of $53.59 \pm 12.87$, while the score after the program varied 
from 64-89 with a mean and standard deviation of $74.47 \pm 8.57$. Regarding to nurses with 30-35 age group, the scores before the program ranged from 3562 with a mean and standard deviation of $46.43 \pm 10.38$, while the score after the program varied from 59-79 with a mean and standard deviation of 67.21 \pm 8.67 . Regarding to nurses with more than 35 years old, the scores before the program ranged from 39-41 with a mean and standard deviation of $40.0 \pm 1.0$, while the scores after the program varied from 54-57 with a mean and standard deviation of $56.0 \pm 1.73$. Overall, the results show that a statistically significant difference was found after implementing the program $(\mathrm{P}<0.001)$ (Table 3$)$.

In the same context, the results before the educational program showed that the nurses with age group between 20-24 got the highest minimum score 49. In addition, the nurses with age group between 25-29 got the highest maximum score (80), while the nurses with more than 35 years old got the least maximum score (41). Moreover, variations were found on the improvement of the nurses' scores after the educational program in all age groups. The highest minimum score (74) was for the nurses with 20-24 age group, and the highest maximum score (89) was for the nurses with 25-29 age group. Consequently, the least minimum and maximum scores (54-57) were for the nurses with more than 35 years respectively. Overall, this study found a statistically significant relationship of this procedure in regards to nurses' age before and after the implementation of the educational program $(\mathrm{p}<0.001)$ (Table 3).

Table 3. The description and relationship between nurses' age groups and implementation of patient care and safety measures for nasogastric tube feeding procedure of the studied nurses $(n=48)$

\begin{tabular}{|c|c|c|c|c|c|c|}
\hline & \multirow[t]{2}{*}{ Total score } & \multicolumn{4}{|c|}{ Age of the nurse } & \multirow[t]{2}{*}{$\mathbf{p}$} \\
\hline & & $\begin{array}{l}20-24 \\
(n=14)\end{array}$ & $\begin{array}{c}25-29 \\
(n=17)\end{array}$ & $\begin{array}{l}30-35 \\
(n=14)\end{array}$ & $\begin{array}{c}\text { Above } 35 \\
(n=3)\end{array}$ & \\
\hline \multirow[t]{6}{*}{ Feeding } & $\begin{array}{c}\text { Before } \\
\text { program }\end{array}$ & & & & & \\
\hline & Min. - Max. & $49.0-59.0$ & $40.0-80.0$ & $35.0-62.0$ & $39.0-41.0$ & \multirow[t]{2}{*}{$0.038^{*}$} \\
\hline & Mean \pm SD. & $53.71 \pm 2.87$ & $\begin{array}{c}53.59 \pm \\
12.87 \\
\end{array}$ & $\begin{array}{c}46.43 \pm \\
10.38 \\
\end{array}$ & $40.0 \pm 1.0$ & \\
\hline & After program & & & & & \\
\hline & Min. - Max. & $74.0-79.0$ & $64.0-89.0$ & $59.0-79.0$ & $54.0-57.0$ & \multirow{2}{*}{$\underset{*}{<0.001}$} \\
\hline & Mean \pm SD. & $77.57 \pm 1.60$ & $74.47 \pm 8.75$ & $67.21 \pm 8.67$ & $56.0 \pm 1.73$ & \\
\hline
\end{tabular}

$\mathrm{p}: \mathrm{p}$ value for comparing between the different studied categories *: Statistically significant at $\mathrm{p} \leq 0.05$ 


\section{The Implementation of Patient Care and Safety Measures regarding Nurses' Qualifications}

Regarding to nurses' with diploma degree, the results show that the nurses' scores before the program varied from 35-59 with a mean and standard deviation of $46.79 \pm 6.92$, while the nurses' score after the program varied from 54-79 with a mean and standard deviation of $69.55 \pm 8.17$. Regarding to the nurses' with a bachelor degree, the scores before the program ranged from 57-68 with a mean and standard deviation of $61.71 \pm 4.03$, while the scores after the program varied from 77-81 with a mean and standard deviation of 78.86 \pm 1.35 . Regarding to nurses with master degree, the scores before the program ranged from 70-80 with a mean and standard deviation of $74.33 \pm$ 5.13 , while the scores after the program varied from 88-89 with a mean and standard deviation of $88.67 \pm 0.58$. Overall, the results show that a statistically significant difference was found before and after implementing the program $(\mathrm{P}<0.001)$ (Table 4).

In the same context, the results before the educational program show that the nurses with a master degree got the highest minimum and maximum scores, i.e., 70-80 respectively, while the nurses with diploma got the least minimum and maximum scores, i.e., 35-59 respectively. Moreover, we found variations of improvement of the nurses' scores after the educational program in the different qualifications. Also, the nurses with a master degree got the highest scores (88-89), while the nurses with diploma got the least scores (5479). Overall, this study found a statistically significant relationship in all steps of this procedure before and after the implementation of the educational program $(\mathrm{p}<0.001)$ (Table 4).

Table 4. The relationship between nurses' qualifications and implementation of patient care and safety measures for each clinical procedure of the studied nurses $(n=48)$

\begin{tabular}{|c|c|c|c|c|c|}
\hline & \multirow[t]{2}{*}{ Total score } & \multicolumn{3}{|c|}{ Qualification of the nurse } & \multirow[t]{2}{*}{$\mathbf{P}$} \\
\hline & & $\begin{array}{l}\text { Diploma } \\
(n=14)\end{array}$ & $\begin{array}{l}\text { Bachelor } \\
(n=17)\end{array}$ & $\begin{array}{l}\text { Master } \\
(n=3)\end{array}$ & \\
\hline \multirow[t]{6}{*}{ Feeding } & Before program & & & & \\
\hline & Min. - Max. & $35.0-59.0$ & $57.0-68.0$ & $70.0-80.0$ & \multirow[t]{2}{*}{$<0.001^{*}$} \\
\hline & Mean \pm SD. & $46.79 \pm 6.92$ & $61.71 \pm 4.03$ & $74.33 \pm 5.13$ & \\
\hline & After program & & & & \\
\hline & Min. - Max. & $54.0-79.0$ & $77.0-81.0$ & $88.0-89.0$ & \multirow[t]{2}{*}{$<0.001^{*}$} \\
\hline & Mean \pm SD. & $69.55 \pm 8.17$ & $78.86 \pm 1.35$ & $88.67 \pm 0.58$ & \\
\hline
\end{tabular}

p: p value for comparing between the different studied categories $\quad *$ : Statistically significant at $\mathrm{p} \leq 0.05$

\section{The Implementation of Patient Care and Safety Measures regarding the Nurses' Experiences}

In regard to nurses with less than five years of experience, the results show that the scores before the program varied from 49-80 with mean and standard deviation of $57.30 \pm 8.38$, while the scores after the program varied 
from 74-89 with a mean and standard deviation of $79.00 \pm 4.23$. In regard to nurses with five to ten years of experience, the scores before the program ranged from 38-62 with mean and standard deviation of $47.24 \pm 8.50$, while the scores after the program varied from 59-79 with mean and standard deviation of $69.12 \pm 6.75$. Regarding to nurses with more than ten years of experience, the scores before the program ranged from 35-41 with mean and standard deviation of $39.0 \pm 1.93$, while the scores after the program varied from 54-62 with mean and standard deviation of $58.63 \pm 2.56$.

In the same context, the results before the educational program show that the nurses with less than five years of experience got the highest minimum and maximum scores (49-80) respectively, while the nurses with more than ten years of experience got the least minimum and maximum scores (35-41) respectively. Moreover, variations of improvement were found in the nurses' scores after the educational program in all categories of experience. The nurses with less than five years of experience got the highest scores (74-89), while the nurses with more than ten years of experience got the least scores (54-62). Overall, the results show that a statistically significant was found before and after implementing the program $(\mathrm{P}<0.001)$ (Table 5).

Table 5. The relationship between nurses' experience and implementation of patient care and safety measures during enteral feeding of the studied nurses $(n=48)$

\begin{tabular}{|c|c|c|c|c|c|}
\hline & \multirow[t]{2}{*}{ Total score } & \multicolumn{3}{|c|}{ Experience of the nurse in ICU } & \multirow[t]{2}{*}{$\mathbf{P}$} \\
\hline & & $\begin{array}{l}\text { Less than } 5 \\
\text { years }(n=14)\end{array}$ & $\begin{array}{l}5-10 \text { years } \\
(n=17)\end{array}$ & $\begin{array}{lr}\text { More } & \text { than } \\
10 & \text { years } \\
(\mathrm{n}=3) & \end{array}$ & \\
\hline \multirow{6}{*}{ Feeding } & Before program & & & & \\
\hline & Min. - Max. & $49.0-80.0$ & $38.0-62.0$ & $35.0-41.0$ & \multirow[t]{2}{*}{$<0.001^{*}$} \\
\hline & Mean \pm SD. & $57.30 \pm 8.38$ & $47.24 \pm 8.50$ & $39.0 \pm 1.93$ & \\
\hline & After program & & & & \\
\hline & Min. - Max. & $74.0-89.0$ & $59.0-79.0$ & $54.0-62.0$ & \multirow[t]{2}{*}{$<0.001^{*}$} \\
\hline & Mean \pm SD. & $79.00 \pm 4.23$ & $69.12 \pm 6.75$ & $58.63 \pm 2.56$ & \\
\hline
\end{tabular}

\section{Discussion}

Nursing care has an important role in an intensive care unit. The nurses' role usually includes clinical assessment, diagnosis, and an individualized plan of expected treatment outcomes for each patient. The ICU staff is responsible for medication, administration, enteral, and parenteral nutrition for patients who cannot eat on their own. ICUs also have respiratory care with specialized training in cardiorespiratory care for critically ill patients (Harrison \& Nixon, 2002; Chaboyer Foster et al., 2004).

Nutritional requirements including enteral nutritional therapy (EN), parenteral nutritional therapy (PN), or combination of both can improve outcomes associated with malnutrition in critically ill patients. Enteral feeding is a common method of maintaining or improving nutritional status in patients 
who are unable to take sufficient nutrition orally (Hejazi Mazloom et al., 2016; Mooi \& Ncama, 2019). Observation of nasogastric tube feeding revealed that feeding was not implemented with safety measures. Moreover, many nurses neglected some items to ensure the patient's safety.

The present results revealed that nurses did not follow the safety measures and infection control criteria regarding the implementing feeding via nasogastric tube to the patients. The majority of nurses in this study did not listen to bowel sounds, Confirm ETT/TT cuff inflation, and irrigate NGT with water. This increases the possibility of patient's complications.

The overall of nurses' performance scores regarding nasogastric tube feeding ranged from 35.0 - 80.0 out of 92 before the educational program with a mean score of $50.69 \pm 10.35$, while that after the educational program ranged from 54-89 with a mean score of $72.10 \pm 9.07$ (Table 2).

Regarding to the nurses' age, the results before the educational program show that the nurses with age group between 20-24 got the highest minimum score 49 out of 92 . In addition, the nurses with age group between 25-29 got the highest maximum score (80), the nurses with age group between 30-35 years got the least minimum score (35), and the nurses with more than 35 years got the least maximum score (41). Moreover, variations of improvement of the nurses' scores were found after the educational program in all age groups. The highest minimum score (74) was for the nurses with 2024 age group, the highest maximum score (89) was for the nurses with 25-29 age group, and the least minimum and maximum scores (54-57) were for the nurses above 35 years respectively. Overall, this study found a statistically significant relationship in all steps of this procedure before and after the implementation of the educational program $(p<0.001)$ (Table 3$)$.

Regarding to the nurses' qualifications, the results before the educational program showed that the nurses with a master degree got the highest minimum and maximum scores (70-80) respectively, while the nurses with diploma got the least minimum and maximum scores (35-59) respectively. Moreover, variations of improvement were found in the nurses' scores after the educational program regarding the different qualifications. The nurses with master degree got the highest scores (88-89), while the nurses with diploma got the least scores (54-79). Overall, this study found a statistically significant relationship in all steps of this procedure before and after the implementation of the educational program $(p<0.001)$ (Table 4).

Regarding to the nurses' experience, the results before the educational program show that the nurses with less than 5 years of experience got the highest maximum and minimum scores (49-80) respectively, while the nurses with more than 10 years of experience got the least minimum and maximum scores (35-41) respectively. 
Moreover, variations of improvement were found in the nurses' scores after the educational program regarding the different years of experience. The highest scores (74-89) were for the nurses with less than 5 years of experience, while the nurses with more than 10 years of experience got the least scores (54-62). Overall, this study found a statistically significant relationship in all steps of this procedure before and after the implementation of the educational program $(\mathrm{p}<0.001)$ (Table 5).

The results of the present study revealed there were variations of improvement regarding to nursing knowledge and practice found after the training program. These findings were supported by Kim and Chang (2019) who found that the studied nurses showed a significant improvement in their perceptions and knowledge of enteral nutrition for ICU patients after the training program. There was a significant improvement in inspecting nostrils daily, flushing the feeding tube before administration, providing medication that needs to be crushed correctly, changing feeding sets, and adjusting feeding schedules. Our findings are similar to Ahmed Ahmed et al. (2018) who reported in her study that there was a highly statistically significant difference $(\mathrm{p} \leq 0.001)$ between total nurse's knowledge level and total practice level at pre implementation of guidelines. Also, there was a statistical significant difference $(\mathrm{P}<0.05)$ between total nurses' knowledge level and total practice level at post implementation of guidelines. Our findings are also parallel with Shahin (2012) who found a significant improvement in knowledge and practice on the studied nurses during enteral feeding for ICU patients after the training program.

Our findings are different from Buccheri Casuccio et al. (2007) who reported that $78.1 \%$ of the nursing staff of two hospitals in Sicily, Italy, has a good knowledge and safe practices concerning food safety and providing feeding through nasogastric tube for critically ill patients.

\section{Conclusion And Recommendations}

In conclusion, this study accessed the effect of an educational program on nurses' practices regarding the implementation of patient care and safety measures in the intensive care units at Al-Assad University Hospital Damascus- Syrian Arab Republic. Our findings highlighted that there was lack and scarcity of nurses' knowledge and performance regarding the nasogastric feeding procedure in this study. The nurses were upset because they didn't follow the protocol due to lack of training programs, too much patients, no enough time, and many other reasons. After going through the program, the nurses were satisfied with the program because there was improvement in their performance, the new guide and protocol. There were actual variations of nurses' knowledge and performance improvement after receiving the program regarding to nurses' age, qualifications, and years of experience. This 
improvement is reflected on patient outcomes and safety. It is recommended to pay more attention to these nurses by helping them to improve their knowledge and practices in all nursing interventions. Furthermore, it is necessary to improve nurses' awareness regarding the quality of care and patient safety. It was also found that the poor knowledge and practice and its complications have a negative impact on the quality of care and patient safety. In addition, nurses with inadequate knowledge and practice often require support in educational programs and additional training for improving their performance to reach the optimal quality of care and patient safety (Shahin, 2012; Bayatmanesh Zagheri Tafreshi et al., 2019).

On the other hand, there were lack of written policies and procedures, lack of in-service training programs to the staff, lack of providing leadership for patient safety initiatives, lack of improving reporting systems and lack of resources, knowledge and practices of the nursing staff (Shahin, 2012; Aziz \& Ali, 2020).

Moreover, regarding to the nurses' qualifications, the nurses with master degree got the highest scores, then the nurses with bachelor, and finally the nurses with diploma got the lowest scores. Regarding to nurses' age, the findings show variations of improvement. The two nurses' age groups 20-24 and 25-29 got the highest scores, while the nurses with more than 35 years got the lowest scores. Regarding to nurses' experiences, the findings of the present study showed that the nurses with less than five years of experience got the highest score, the nurses with five to ten years of experience, and finally the nurses with more than ten years of experience got the lowest average score.

Nursing staff should focus on participating and attending the educational and training programs to improve their knowledge and performance regarding all the necessary skills and procedures needed in the critical care units. This is aimed at providing a high quality of care for critically ill patients and to also improve patient safety. By improving these methods and giving much more educational and training support, the quality of care and safety can be improved (Bourgault Ipe et al., 2007; Ahmed Ahmed et al., 2018).

Based on the findings of the present study, the following recommendations are suggested:

- $\quad$ Hospitals should have the updated checklists and guidelines for all procedures.

Hospitals must create educational and training programs for all medical staff to follow the standards and make them up to date.

Hospitals should clearly communicate patient-safety requirements and enforce those standards. 
- $\quad$ Professional associations, colleges, and hospital associations should promote improved patient safety by disseminating information on best practices and giving professional training in risk management.

\section{References:}

1. Ahmed, F., et al. (2018). "Effect of Educational Nursing Guidelines Regarding Enteral Feeding on Nurses' Knowledge and Practices at Critical Care Units." IOSR Journal of Nursing and Health Science (IOSR-JNHS) 7(5): 69-75.

2. Alhawsawi, A., et al. (2017). Essentials of patient safety, Riyadh, Saudi Arabia: Saudi Commission for Health Specialties.

3. Alotaibi, Y. K. \& Federico, F. (2017). "The impact of health information technology on patient safety." Saudi medical journal 38(12): 1173.

4. Anderson, L. (2019). "Enteral feeding tubes: an overview of nursing care." British Journal of Nursing 28(12): 748-754.

5. Ang, S. Y., et al. (2020). "A Descriptive Study of enteral tube feeding among adults in an acute care tertiary hospital-patient selection, characteristics and complications." Clinical nutrition ESPEN 37: 5864.

6. Australia, C. O. I. C. M. O. \& Zealand, N. (2010). "Minimum standards for intensive care units."

7. Aziz, K. M. \& Ali, S. A. (2020). "Determination of the Critical Care Nurses Knowledge Toward Enteral Tube Feeding in AL-Hilla Teaching Hospitals (Interventional study)." Medico Legal Update 20(1): 1153-1157.

8. Bayatmanesh, H., et al. (2019). "Patient Safety Observation by Nurses Working in the Intensive Care Units of Selected Hospitals Affiliated to Yasuj University of Medical Sciences." ISMJ 21(6): 493-506.

9. Blumenstein, I., et al. (2014). "Gastroenteric tube feeding: techniques, problems and solutions." World journal of gastroenterology: WJG 20(26): 8505.

10. Boullata, J. I., et al. (2017). "ASPEN safe practices for enteral nutrition therapy." Journal of Parenteral and Enteral Nutrition 41(1): 15-103.

11. Bourgault, A. M., et al. (2007). "Development of evidence-based guidelines and critical care nurses' knowledge of enteral feeding." Critical care nurse 27(4): 17-29.

12. Bower, R. H. (1990). "Nutritional and metabolic support of critically ill patients." JPEN 14(5 supply): 257S259S.

13. Btaiche, I. F., et al. (2010). "Critical illness, gastrointestinal complications, and medication therapy during enteral feeding in critically ill adult patients." Nutrition in Clinical Practice 25(1): 32-49. 
14. Buccheri, C., et al. (2007). "Food safety in hospital: knowledge, attitudes and practices of nursing staff of two hospitals in Sicily, Italy." BMC health services research 7(1): 1-11.

15. Camiré, E., et al. (2009). "Medication errors in critical care: risk factors, prevention and disclosure." Cmaj 180(9): 936-943.

16. Chaboyer, W., et al. (2004). "The intensive care unit liaison nurse: towards a clear role description." Intensive and Critical Care Nursing 20(2): 77-86.

17. Ciocon, J. O., et al. (1988). "Tube feedings in elderly patients: indications, benefits, and complications." Archives of internal medicine 148(2): 429-433.

18. Gavi, S., et al. (2008). "Management of feeding tube complications in the long-term care resident." Annals of long term care 16(4): 28.

19. Harrison, L. \& Nixon, G. (2002). "Nursing activity in general intensive care." Journal of Clinical Nursing 11(2): 158-167.

20. Hejazi, N., et al. (2016). "Nutritional assessment in critically ill patients." Iranian journal of medical sciences 41(3): 171.

21. Kim, H. \& Chang, S. J. (2019). "Implementing an educational program to improve critical care nurses' enteral nutritional support." Australian Critical Care 32(3): 218-222.

22. Marshall, A. P., et al. (2012). "Optimizing nutrition in intensive care units: empowering critical care nurses to be effective agents of change." American journal of critical care 21(3): 186-194.

23. Marshall, J. C., et al. (2017). "What is an intensive care unit? A report of the task force of the World Federation of Societies of Intensive and Critical Care Medicine." Journal of critical care 37: 270-276.

24. McLaren, S. \& Arbuckle, C. (2020). "Providing optimal nursing care for patients undergoing enteral feeding." Nursing standard 35(3): 6065.

25. Metheny, N. A., et al. (2007). "Complications related to feeding tube placement." Current opinion in gastroenterology 23(2): 178-182.

26. Mooi, N. M. \& Ncama, B. P. (2019). "Evidence on nutritional therapy practice guidelines and implementation in adult critically ill patients: A systematic scoping review." Curationis 42(1): 1-13.

27. Organization, W. H. (2020). Development of a draft global patient safety action plan, World Health Organization. Regional Office for the Eastern Mediterranean.

28. Persenius, M. W., et al. (2006). "Enteral nutrition in intensive care: nurses' perceptions and bedside observations." Intensive and Critical Care Nursing 22(2): 82-94.

29. Rungta, N., et al. (2010). "ICU planning and designing in Indiaguidelines 2010." ISCCM Guidelines Committee. 
30. Scolapio, J. S. (2007). "Decreasing aspiration risk with enteral feeding." Gastrointestinal endoscopy clinics of North America 17(4): 711-716.

31. Serna, E. D. \& McCarthy, M. S. (2006). "Heads up to prevent aspiration during enteral feeding." Nursing2020 36(1): 76-77.

32. Shahin, M. A. (2012). "Impact of a Designed Instructional Program about Enteral Nutrition on the Nurses' knowledge and Practices at the Critical Care Department of Almanial University Hospital." CU Theses.

33. Strollo, B. P., et al. (2017). "Complications of home enteral nutrition: mechanical complications and access issues in the home setting." Nutrition in Clinical Practice 32(6): 723-729.

34. Thompson, J. M. (1997). "Mosby's clinical nursing."

35. Vaithiswaran, V., et al. (2010). "Effect of early enteral feeding after upper gastrointestinal surgery." Tropical Gastroenterology 29(2): 9194.

36. Wiegand, D. L. (2013). AACN Procedure Manual for Critical Care-EBook, Elsevier Health Sciences. 